\title{
Research on Advanced Distributed Learning by Using SCORM
}

\author{
Ronghui $\mathrm{Wu}^{1}$, Renfa $\mathrm{Li}^{1}$, Fei $\mathrm{Yu}^{1,2,3}$, Guangxue Yue ${ }^{1}$, and Chen $\mathrm{Xu}^{1,2,3}$ \\ ${ }^{1}$ College of Computer \& Communication, Hunan University, Changsha 410082, China \\ wrh@hnu.cn \\ 2 Jiangsu Provincial Key Laboratory of Computer Information Processing Technology, \\ Province, Suzhou University, Suzhou, 2150063, China \\ hunanyufei@126.com \\ ${ }^{3}$ Guangdong Province Key Lab of Electronic Commerce Market Application Technology, \\ Guangdong University of Business Studies, Guangzhou,510320, China
}

Yufei@hunau.edu.cn

\begin{abstract}
The Sharable Content Object Reference Model (SCORM) was created to assist the e-learning industry in standardizing the best approaches to creating, storing, transferring and deploying learning content. The Advanced Distributed Learning Initiative(ADL) provides these guidelines consequent to a survey of authoring tools that support SCORM. In this paper, we us SCORM Sequencing Definition model to construct adaptive courseware. At first introduces the architecture of the whole system; Then introduces the way to build adaptive courseware, Finally gives a conclusion.
\end{abstract}

\section{Introduction}

The SCORM introduces technical changes in the way authoring tools prepare and package learning content. Beyond the technical changes, the SCORM affects the overall relationship between authoring tools and other key e-learning elements, such as Learning Management Systems (LMSs) and content repositories. This paper is primary to provide insights to authoring tool vendors about key aspects of SCORM.

\section{Architecture}

Before we utilize SCORM into our courseware, we should make a brief cognition of how it works. As we described in section 1, SCORM 1.3 is mainly consisted of two parts: SCORM Content Aggregation Model and SCORM Run-Time Environment.

\subsection{SCORM Content Aggregation Model}

SCORM Content Aggregation Model: not only defines the content components of a learning experience but also how to represent the intended behavior of a learning experience (Content Structure) and how to aggregate activities of learning resources 
for movement between different environments (Content Packaging). Furthermore, it provides a mechanism for describing specific instances of the components of the content model named meta-data. The basic content objects in SCORM are categorized as Asset and Sharable Content Object (SCO).

The SCORM Version 1.2 Content Aggregation Model, describes three types of SCORM Meta-data: Content Aggregation, SCO and Asset. Any or all of these three types may be contained in two different XML documents: as standalone meta-data (i.e., a single XML document that describes one thing), or embedded in a SCORM manifest. A manifest allows for meta-data to be represented in two ways: in-line or referenced to a file. Meta-data that is part of a manifest is referred to as "in-line" meta-data in the SCORM Content Packaging Information Model.

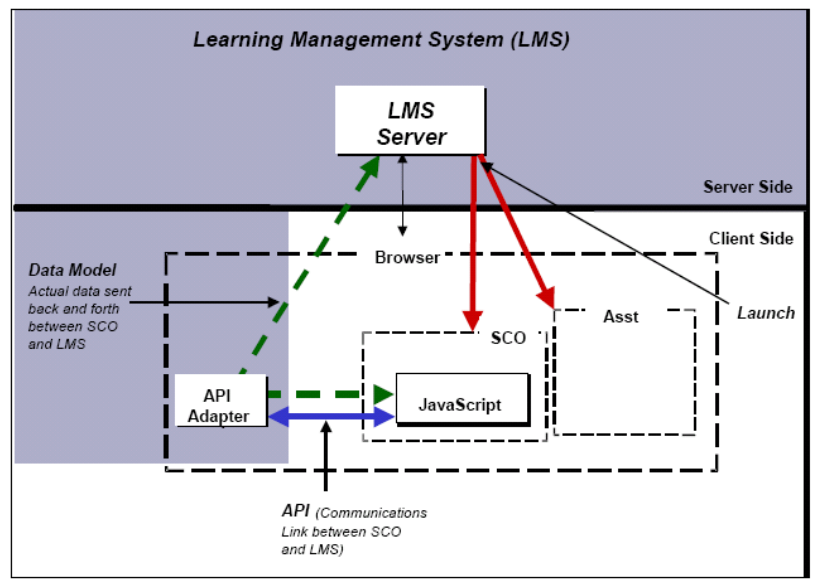

Fig. 1. Shows the system architecture of the SCORM Run-Time Environment and demonstrates how the system is worked

\subsection{SCORM Run-Time Environment}

SCORM Run-Time Environment defines a common content object launch mechanism, a common communication mechanism between content objects and LMSs, and a common data model for tracking a learner's experience with content objects.

In SCORM 1.3, the SCORM RTE is constructed based on a JSP\&Java Servletenabled jakarta-tomcat-4.1.24 Servlet Engine and Web server. On the server side, a JSP component is used to dynamically analysis and render the content aggregation file of one specific courseware into a navigation menu which will appears as a series of hyperlinks whose targets contain the corresponding launch locations of SCOs. What's more, there are also several Java Servlet components that are responsible for controlling actual sequencing of SCOs and handling communication between RTE and SCO. While on the client side, a non-face Java Applet is implement as the SCORM RTE API Adapter, which provides the communication to the RTE serverside Servlet components. 


\section{Construct the Adaptive Courseware}

While the ADL provides several useful SCORM utilities and testing tools that are freely available to the public, ADL does not provide a means to directly test authoring tools for SCORM conformance. The following step is to design the activity tree of the whole courseware to achieve the goal of adaptive capacity. Fig. 2 shows our design of activity tree of CIS courseware.

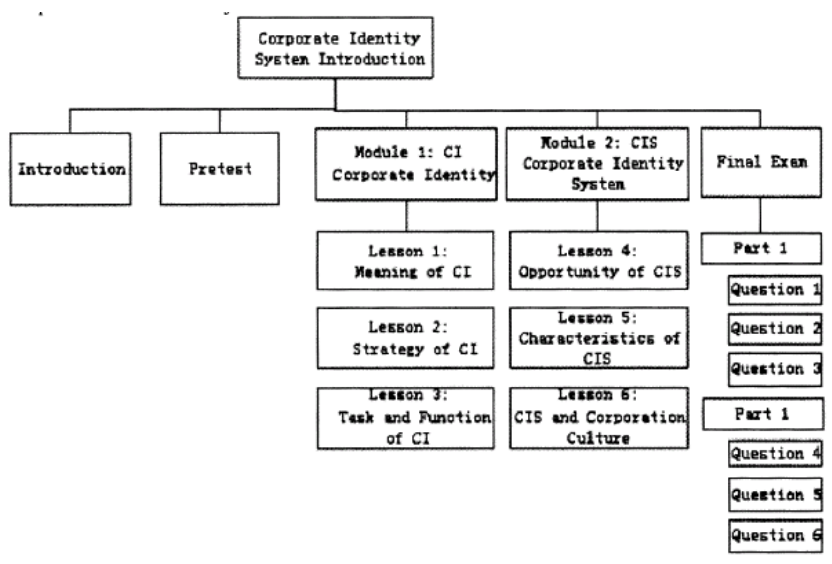

Fig. 2. The activity tree of the CIS courseware

\section{System Analysis}

The adaptive capacity of a Web-based course is probably the next main issue of the individuals and organizations using E-learning standards to develop coursewares after achieving the goal of reusability and interoperability of the coursewares. This paper introduces a method of constructing courseware by using SCORM Sequencing Model, and shows how to use this model to define required content organizations.

Being conformant with the E-learning standard, it can be reusable and interoperable through the internet which greatly meets the need of exchanging some newly accumulated experience between different organizations. By using the SCORM Sequencing Definition Model, the courseware realizes the adaptive capacity, that is different learners view different content according to their acknowledge level.

\section{Conclusion}

By using the Sequencing Definition Model, we define more complicated organization of the courseware with the adaptive feature according to various instructional needs. Furthermore, we plan to develop a SCORM-conformant intelligent tutoring system to improve the adaptive characteristics of our Web-based coursewares. 


\section{References}

[1] Charlton J P,Birkett P E. : An Integrative Model of Factors Related to Computing Course Performance.Education Computing Research,1999,20(3) :237 257

[2] Valverde-Albacete, F.J. Pedraza-Jimenez, R, Cid-Sueiro, J. : An environment for instructional content design based on competences.Proceedings of the ACM Symposium on Applied Computing, 2003

[3] Jun-Ming Su,Shian-Shyong Tseng,Jui-Feng Weng. : An Object Based Authoring Tool for Creating SCORM Compliant Course. Proceedings of Advanced Information Networking and Applications(AINA'2005) 